\title{
COMPREENSÃO DOS COORDENADORES DE SERVIÇOS DE SAÚDE SOBRE EDUCAÇÃO PERMANENTE
}

\author{
Maria de Lourdes Custódio Duarte ${ }^{1}$, Aládia Inês de Oliveira²
}

\begin{abstract}
RESUMO: O estudo objetivou compreender o conhecimento dos coordenadores dos serviços de saúde de um município do Rio Grande do Sul sobre Educação Permanente. Participaram quatro enfermeiros, um psicólogo e um odontólogo, coordenadores de serviços; os dados foram coletados por meio de entrevista semiestruturada em novembro de $2011 \mathrm{e}$ analisados seguindo os passos da analise temática. Da análise emergiram duas categorias: Compreensão sobre a Educação Permanente e Desafios para a Implantação da Política de Educação Permanente no município. Salienta-se a importância dos coordenadores buscarem conhecimento sobre essa política ministerial com vistas a mudanças nas práticas de saúde do município e das práticas de formação dos profissionais, com vistas a atender princípios do Sistema Único de Saúde.

PALAVRAS-CHAVE: Educação em saúde; Gestão em saúde; Recursos humanos em saúde.
\end{abstract}

\section{HEALTH SERVICES COORDINATORS' UNDERSTANDING ABOUT CONTINUING EDUCATION}

\begin{abstract}
The study aimed to investigate what the health service coordinators in a municipality in the state of Rio Grande do Sul knew about continuing education. The service coordinators who participated were four nurses, one psychologist and one dentist; the data was collected through semi-structured interviews in November 2011 and was analyzed following the steps of thematic analysis. Two categories emerged from the analysis: Understanding about Continuing Education and Challenges for the implantation of the Continuing Education Policy in the municipality. It stands out that it is important for the coordinators to seek knowledge about this ministerial policy with a view to changes in the health practices in the municipality, and in the practices involved in training health professionals so as to better attend the Unified Health System's principles.
\end{abstract}

KEYWORDS: Health education; Health management; Human resources in health.

\section{COMPRENSIÓN DE LOS COORDINADORES DE SERVICIOS DE SALUD ACERCA DE LA EDUCACIÓN PERMANENTE}

RESUMEN: El estudio tuvo la finalidad de comprender el conocimiento de los coordinadores de los servicios de salud de un municipio de Rio Grande do Sul acerca de la Educación Permanente. Participaron cuatro enfermeros, un psicólogo y un odontólogo, coordinadores de servicios; los datos fueron obtenidos por medio de entrevista semiestructurada en noviembre de 2011 y analizados de acuerdo con los pasos del análisis temático. Del análisis emergieron dos categorías: Comprensión sobre la Educación Permanente y Desafíos para la implantación de la Política de Educación Permanente en municipio. Se destaca la importancia de la búsqueda del conocimiento sobre esa política ministerial por los coordinadores a fin de cambiar las prácticas de salud del municipio y las prácticas de formación de los profesionales, para atender a principios del Sistema Único de Salud. PALABRAS CLAVES: Educación en salud; Administración en salud; Recursos humanos en salud.

${ }^{1}$ Enfermeira. Mestre em Enfermagem Psiquiátrica e Ciências Humanas. Doutoranda pelo Programa de Pós-Graduação em Enfermagem da Universidade Federal do Rio Grande do Sul - UFRGS. Professora da Universidade Federal do Pampa UNIPAMPA - RS.

${ }^{2}$ Bacharel em História. Especialista em Gestão do Trabalho. 


\section{INTRODUÇÃO}

Nos últimos anos, o sistema de saúde brasileiro tem passado por intensas transformações, instituído legalmente pela Constituição Federal de 1988 e pela implantação do Sistema Único de Saúde (SUS), em 1990. Nesse contexto, a construção de novas práticas de saúde tem se configurado como um desafio devido às dificuldades de superação de um modelo biologicista e mecanicista, por outro mais amplo, voltado à integralidade, humanização e inclusão da participação dos trabalhadores em saúde e usuários ${ }^{(1)}$.

Nesse cenário, em 2003 o Ministério da Saúde (MS) criou a Secretaria de Gestão do Trabalho e da Educação na Saúde (SGTES), a qual assumiu o papel definido na legislação, de gestor federal do SUS no que diz respeito à formulação das políticas orientadoras da formação, desenvolvimento, distribuição, regulação e gestão dos trabalhadores da saúde, no Brasil ${ }^{(2)}$. Dessa forma, criou-se espaço de discussão sobre as diretrizes para a formulação de políticas de formação e educação permanente dos trabalhadores da área de saúde.

Em 2004, o MS implantou a Política Nacional de Educação Permanente em Saúde (PNEPS), por meio da Portaria GM/MS n. 198, substituída posteriormente pela Portaria GM/MS n. 1.996 de $2007^{(3)}$, superando a lógica da Educação Continuada que perpetuava na área da saúde e que muito era criticada por pautar-se em uma educação parcelada, fora do contexto real das necessidades de saúde e por favorecer o atendimento de determinadas profissões hegemônicas ${ }^{(2,4-5)}$. No entanto, a Educação Permanente em Saúde parte do princípio que o conteúdo a ser estudado deve ser gerado a partir de dúvidas e necessidades de conhecimento emergidas em situações vivenciadas pelos próprios profissionais ${ }^{(5-7)}$.

Essa Política prevê a criação dos Pólos de Educação Permanente em Saúde para o SUS (PEPS) para que se configurem como espaços de formulação de políticas e de negociação orientadas pelas necessidades de formação e desenvolvimento de profissionais de saúde pelos princípios e diretrizes do SUS. Cada PEPS será referência e se responsabilizará por um determinado território, sendo que este terá a responsabilidade de apresentar suas necessidades de formação e desenvolvimento ${ }^{(2)}$. Assim, a Educação Permanente passa a ser vista como uma estratégia fundamental para a mudança no sistema de saúde e para a recomposição das práticas de formação, atenção, gestão, formulação de políticas e controle social no setor saúde. Ela deixa de se restringir à formação profissional e passa a atuar de forma articulada entre instituições formadoras, gestores do SUS, serviços, instâncias do controle social e representações estudantis como dispositivo para a mudança nas práticas de saúde e mudança nas práticas de formação em saúde ${ }^{(8-9)}$.

O interesse por este tema surgiu através de observações em uma Secretaria Municipal de Saúde de um município de pequeno porte do Rio Grande do Sul, no qual constatou-se a falta de uma Política de Educação Permanente. Foi evidenciado nesse município o predomínio de práticas de saúde fragmentadas, serviços de saúde desarticulados e profissionais de saúde que desenvolvem práticas de saúde desvinculadas do SUS. Somado a isso, percebeu-se que muitos gestores de saúde desconhecem a Política de Educação Permanente e ainda confundiam-na com Educação Continuada. Assim, entende-se que a falta de conhecimento dos gestores de saúde sobre a Política de Educação Permanente contribui para a manutenção dessas práticas.

A grande relevância da Educação Permanente em Saúde fez com que a mesma se tornasse estratégia fundamental do SUS para a formação e o desenvolvimento de trabalhadores para a saúde, na perspectiva de aumentar a qualidade dos serviços prestados e o fortalecimento do controle social no SUS. Então, questiona-se: Qual a compreensão dos gestores de saúde sobre Educação Permanente?

Acredita-se que este estudo pode contribuir para sensibilizar os coordenadores dos serviços de saúde do município sobre a importância da implantação da PNEPS para o desenvolvimento profissional, nas transformações das práticas de saúde e na organização dos serviços.

Portanto, foram objetivos desta pesquisa compreender o conhecimento dos coordenadores dos Serviços de Saúde sobre a Educação Permanente e identificar os principais desafios na implantação dessa política no município.

\section{MÉTODO}

Este estudo tem caráter exploratório-descritivo, com abordagem qualitativa ${ }^{(10)}$, sendo realizado em um município de 24 mil habitante do interior do Estado do Rio Grande do Sul. Os sujeitos do estudo compreenderam a totalidade dos coordenadores dos serviços de saúde do município, ou seja, quatro enfermeiros, um psicólogo e um odontólogo, totalizando seis entrevistados. O tipo da amostra foi intencional por convite, tendo como critério de inclusão aqueles coordenadores que estivessem em pleno exercício da função no período da coleta de dados e por critério de exclusão os que estivessem afastados do trabalho por motivo de 
licença saúde ou férias. A coleta dos dados ocorreu em novembro de 2011, por meio de entrevistas individuais semiestruturadas, consistindo de três questionamentos: Qual é a sua compreensão sobre a Educação Permanente? Existem desafios para a implantação da Política de Educação Permanente no município? Quais?

Procedeu-se à leitura integral das entrevistas, adotando-se o referencial de análise temática para apreciação crítica do conteúdo, buscando-se encontrar os trechos significativos para a constituição dos temas abordados em obras pesquisadas, em relação ao objetivo do estudo. Seguindo-se os passos de pré-análise e exploração do material sendo possível a organização e leitura repetida do corpus de pesquisa. Posteriormente, procedeu-se ao tratamento e interpretação dos resultados obtidos, descritos em unidades de registro e de contexto, que permitiram o agrupamento de ideias relevantes em categorias ${ }^{(10)}$, representadas por dois eixos temáticos de discussão, a saber: Compreensão sobre Educação Permanente e Desafios para a implantação da Política de Educação Permanente no municipio.

Os sujeitos que compuseram a amostra do estudo foram numerados de um a seis na ordem cronológica da realização das entrevistas e denominados de "E" para os Enfermeiros, "P" para Psicólogo e "O" para Odontólogo.

As entrevistas foram pré-agendadas e os dados coletados em uma sala reservada no próprio local de trabalho dos coordenadores de serviço, com tempo de duração em torno de 45 minutos. A pesquisa foi aprovada pelo Comitê de Ética da Universidade Federal do Pampa, conforme protocolo de número 043/2011, sendo considerados os aspectos éticos, envolvendo seres humanos, em conformidade com o previsto na Resolução 196/96 do Conselho Nacional de Saúde ${ }^{(11)}$. Os participantes assinaram o Termo de Consentimento Livre e Esclarecido após terem sido informados sobre os objetivos da pesquisa.

\section{RESULTADOS}

\section{Compreensão sobre Educação Permanente}

Esta categoria dedica-se a apresentar e analisar os dados coletados que remetem a compreensão sobre a Educação Permanente na perspectiva dos coordenadores dos serviços de saúde do município estudado. Essas compreensões permeiam os seguintes temas, a seguir: a) atualização através de seminários, reuniões e encontros; b) uma construção envolvendo todos os atores sociais e c) processo de transformação das práticas profissionais.

Os coordenadores relatam que a Educação Permanente é a oportunidade de se atualizar, através da participação em seminários, reuniões, capacitações e encontros, com o objetivo de qualificação, visando atender as demandas da comunidade e do próprio serviço, a partir de questões do cotidiano do próprio trabalho. Esse conhecimento adquirido nas capacitações, na visão dos entrevistados, é perpetuado a outras pessoas que usufruem do conhecimento, como recortes das falas:

Acho que é a oportunidade de estar se reunindo, de ir a encontros, de poder fazer estudo a respeito do dia-adia do trabalho, de coisas novas que possa surgir, mas através de seminários, de discussões e estudos. (E1)

É aquele conhecimento que a gente passa para alguém e essa pessoa adquire ele e continua usufruindo esse conhecimento pro resto de sua vida. $(\mathrm{O} 4)$

Alguns entrevistados entendem que a Educação Permanente é uma construção envolvendo todos os atores sociais, salientando que essa Política realmente seja permanente, ou seja, que tenha continuidade.

Educação Permanente não é algo dado, ela é um construir, justamente com os atores sociais envolvidos na construção de uma educação. Ela deve ser permanente, que não tenha prazo pra acabar e que tenha uma continuidade. $(\mathrm{P} 2)$

Educação Permanente pra mim é o trabalho em equipe, são as trocas entre as equipes multidisciplinares, dos diversos segmentos, tanto dos gestores quanto dos profissionais técnicos, controle social e comunidade, com o principal objetivo de mudar as nossas práticas profissionais, beneficiando os usuários. (E6)

A Educação Permanente é percebida por um coordenador como um subsídio de aprimoramento do trabalho, provocando a autocrítica das pessoas envolvidas e consequentemente transformando as práticas dos profissionais:

A Educação Permanente vai te dar subsidio para aprimorar o teu trabalho, fazendo uma autocrítica para conseguir mudar a tua prática. Ver aquilo que não tá funcionando direito. (E5) 


\section{Desafios para a implantação da Política de Educação Permanente no município}

Nesta categoria são expostos os desafios trazidos nas falas dos entrevistados sobre a implantação da Política de Educação Permanente no município. Esses desafios permeiam os seguintes assuntos: a) grande rotatividade de profissionais; b) resistência dos profissionais e gestores; c) falta de conhecimento sobre a política e d) falta de participação do controle social.

Apresenta-se abaixo recortes de depoimentos que exemplificam esses desafios:

Um desafio que eu percebo aqui é que tem muita rotatividade de profissionais. (P2)

Percebo a resistência de muitas pessoas que já vem há muito tempo trabalhando de uma mesma forma, tanto dos gestores quanto dos profissionais. (E1)

Para um coordenador de serviço o grande desafio no município é a falta de conhecimento dos profissionais e gestores sobre a Educação Permanente, no entanto percebe-se que o grande desafio é superar a fragmentação da própria equipe, ou seja, de um lado os que têm nível superior e do outro, os de nível médio.

É algo ainda que as pessoas ainda tem que conseguir entender e como fazer né, porque até nós que somos servidores qualificados temos as vezes dúvidas do que seja uma Educação Permanente, uma Educação Continuada. Então, imagina as pessoas que tão ali, os técnicos, os auxiliares de conseguir sua importância. (P2)

Outro desafio percebido para a implantação da PEPS no município é a falta de participação da comunidade e do próprio controle social na identificação e na reivindicação das suas necessidades, como recorte abaixo:

Não contamos com a participação da comunidade na definição das suas próprias necessidades. $O$ controle social é pouco participativo. (E6)

\section{ANÁLISE E DISCUSSÃO}

\section{Compreensão sobre Educação Permanente}

O conjunto das falas permite considerar que alguns coordenadores do município estudado desconhecem o conceito de Educação Permanente e que verbalizam nas entrevistas propostas da Educação Continuada fundamentadas na atualização técnico-científica e na transmissão do conhecimento, como sendo Educação Permanente. Assim, enfatiza-se a repercussão que essa confusão de conceitos tem em um município de pequeno porte, tendo em vista que esses profissionais são coordenadores dos serviços de saúde e que, por si só, são formadores de opinião.

A Educação Continuada caracteriza-se pelo enfoque em temas, é praticada de forma fragmentada e voltada apenas para a atualização técnico-científica. Utiliza-se da pedagogia da transmissão e memorização de conhecimentos, o que contribui para a reprodução dos modelos hegemônicos, ao invés de favorecer mudanças significativas das práticas, da gestão e do controle social ${ }^{(4)}$. Somado a isto, as capacitações não se mostram eficazes para possibilitar a incorporação de novos conceitos e princípios às práticas estabelecidas por trabalharem de maneira descontextualizada e se basearem principalmente na transmissão de conhecimentos ${ }^{(9)}$.

A ação da Educação Permanente em Saúde não se trata de capacitações ou de treinamentos, mas da construção de conhecimentos numa vinculação horizontal, intersetorial e interdisciplinar. Prioriza a relação ensinoaprendizagem movida pelo debate crítico e discussões das exigências presentes no cotidiano dos serviços de saúde, o que significa que o ponto de partida são os problemas ou a problematização da realidade concreta ${ }^{(12)}$.

A Educação Permanente é entendida pelos participantes como sendo um trabalho em equipe multidisciplinar, fundamentada nas trocas entre gestores, profissionais de saúde e o controle social, com o objetivo de transformar as práticas profissionais, na qual o usuário deve ser o maior beneficiado. Assim, essa política surge como uma estratégia de inclusão de todos os atores sociais envolvidos na construção de um sistema de saúde de qualidade, pautado na integralidade das ações. Os trabalhadores de saúde passam a ser reconhecidos como sujeitos do processo de trabalho no qual estão inseridos e não mais como meros executores de políticas e programas de saúde.

A Educação Permanente deixa de se restringir à formação profissional sendo vista como uma estratégia fundamental para a recomposição das práticas de formação, atenção, gestão, formulação de políticas e controle social no setor da saúde. Nessa perspectiva, pode-se afirmar que a PEPS congrega, articula e coloca em roda diferentes atores, destinando a todos um lugar de protagonismo na condução de sistemas locais de saúde ${ }^{(8)}$. 
A Educação Permanente é apresentada pelo MS como estratégia de reestruturação dos serviços, a partir da análise dos determinantes sociais e econômicos, mas, sobretudo, de valores e conceitos dos profissionais. Propõe transformar o profissional em sujeito, colocando-o no centro do processo ensino aprendizagem $^{(4)}$. Assim, entende-se que essas ideias devem ser compreendidas e assimiladas por todos os sujeitos envolvidos a começar pelos profissionais dos serviços de saúde local. As mudanças só irão acontecer de forma efetiva no momento em que os vários agentes sociais assumirem o seu papel de sujeitos na transformação das práticas profissionais e na organização dos serviços de saúde ${ }^{(13)}$.

Percebeu-se nesta categoria, a confusão de conceitos nas falas de alguns coordenadores, uma vez que utilizam definições e estratégias da Educação Continuada, como se fosse Educação Permanente. No entanto, a grande maioria dos entrevistados percebe a importância a Educação Permanente como uma política que envolve todos os atores sociais, visando o processo de transformação das práticas profissionais, além de servir como um instrumento valioso para a qualificação do trabalho prestado à comunidade.

\section{Desafios para a implantação da Política de Educação Permanente no município}

Os recursos humanos no setor saúde constituem a base para a viabilização e implementação dos projetos, das ações e serviços de saúde disponíveis para a população. A utilização dos avanços tecnológicos e da alta tecnologia não substituirá a atuação de um profissional de saúde na função essencial de atendimento àqueles que necessitam de atenção. Assim, sabe-se que o investimento nos recursos humanos irá influenciar decisivamente na melhoria dos serviços de saúde prestados a população ${ }^{(14)}$.

A Reforma Administrativa do Estado Brasileiro nos anos 90 abandona a ideia do Estado mínimo e concentra sua atenção na reforma do Estado, impulsionando a adoção de relações trabalhistas precárias no momento em que ocorria a descentralização do SUS. O enorme incremento do número de postos de trabalho na saúde pública dos municípios, acompanhado das restrições jurídico-legais, como a Lei de Responsabilidade Fiscal ${ }^{(15)}$, propulsionaram a adoção de diversas formas de contratação. Na área da saúde, essa flexibilização dos vínculos trabalhistas ocasionou rotatividade dos profissionais e descontinuidade da as- sistência rompimento do vínculo entre o profissional e a população. Com vínculos não-estáveis, os profissionais ficam sujeitos à instabilidade política, a lei da oferta e da procura e à disputa predatória entre os municípios, o que compromete a sua dedicação ${ }^{(16)}$.

A criação SGTES, em 2003, representou um novo rumo para a área de Recursos Humanos, pois a partir desse momento esta secretaria passa a tratar especificamente da gestão dos recursos humanos na dimensão do trabalho e da educação na saúde. Com esta ação política, o MS tomou para si a responsabilidade de produzir a maior transformação, no que diz respeito à formulação das políticas orientadoras da gestão, formação, qualificação e regulação dos trabalhadores de saúde no Brasil. Pensar hoje a questão dos recursos humanos significa pensar aqueles que são os protagonistas da reforma sanitária, aqueles que detêm a técnica, o saber e o trabalho a serem postos a favor da população brasileira ${ }^{(17)}$.

A resistência dos profissionais de saúde em relação a Educação Permanente está associada ao fato de que os profissionais atualmente em atividade, foram formados a partir de uma visão tecnicista e especializada, que privilegia a atuação centrada no modelo médico-hospitalar, baseada, principalmente, nos aspectos biológicos e tecnológicos da assistência. Por isso, os profissionais vêm enfrentando dificuldades para lidar com questões amplas da atuação (prática) no SUS, como, por exemplo, trabalhar em equipe, desenvolver uma atuação conjunta com a família e com a comunidade, produção de subjetividades e habilidades técnicas e de pensamento ${ }^{(18)}$.

No âmbito da gestão, também podemos associar a resistência dos gestores ao modelo de gestão vigente, verticalizado e centralizado, o que impossibilita a implementação de ações de interdisciplinaridade, intersetorialidade, o trabalho em equipe e a autonomização dos sujeitos. Dessa forma, o estilo de gestão autoritário não deveria ser adotado nos serviços públicos de saúde, pois além de não reconhecer o papel central dos profissionais, a sua capacidade de promover o comprometimento e envolvimento destes com os objetivos da Educação Permanente torna-se limitada. Ou seja, afastando-os das atividades que não sejam eminentemente técnicas, não gerando a sinergia que ocorre quando há participação plena de todos os atores envolvidos no processo ${ }^{(19)}$.

Percebe-se, por meio do recorte da fala do coordenador, anteriormente apresentado, que este coloca-se em uma posição de superioridade e de maior qualificação que aos demais profissionais, o que certamente reflete 
em uma prática segmentada no cotidiano de trabalho. Enfatiza-se ainda, que na PEPS, todos os profissionais assumem a mesma importância, não havendo distinções entre o nível de escolaridade desses protagonistas.

Constata-se que estes desafios se devem a manutenção de ações e práticas de saúde parceladas, desarticuladas ao modelo de saúde vigente, verticalizado, centralizado e autoritário. A formação dos profissionais pode ser considerada outro aspecto preocupante neste desenho foi construída nos bancos escolares de forma fragmentada e distante do perfil adequado para o trabalho na área da saúde pública ${ }^{(6)}$.

A PEPS é uma política transversal, que pressupõe o envolvimento do conjunto de atores do SUS, dos vários segmentos da formação, da atenção, da gestão e do controle social no enfrentamento dos desafios coletivos ora postos na implementação da política no Estado $^{(2)}$. Ainda, esse é um processo em construção no Estado a partir de 2003, que demandará tempo, enfrentamento das barreiras culturais e paciência, para se ver os resultados desse processo

Em relação à participação popular e o controle social, percebe-se, na fala de um coordenador, que são incipientes no município, não havendo envolvimento com a tomada de decisões relativas as necessidades de saúde da própria comunidade. No Brasil, apesar da população ter assento nas instâncias de controle social nas três esferas do governo, garantida pela Constituição Federal de $1988^{(20)}$ é possível perceber que ainda é grande a distância entre a construção dos direitos e o seu efetivo exercício pela população. Por isso o mecanismo de controle social precisa ser aperfeiçoado e consolidado, a partir do "empoderamento" da população que, assim, estará cada vez mais ciente de sua co-participação na direção da melhoria da qualidade de vida.

Os preceitos da PNEPS caracterizam como fundamental a participação dos vários segmentos do SUS, destacando a importância do controle social, nas estratégias sugeridas pela política, como as Comissões da Integração Ensino Serviço, os Colegiados de Gestão Regional e trabalhar na perspectiva da elaboração de Planos de Ação Regional de Educação Permanente em Saúde( ${ }^{(2)}$.

Constatou-se nesta categoria que ainda há uma série de desafios a serem superados no município para a implementação da PEPS, como a grande rotatividade de profissionais, resistência dos profissionais e gestores, falta de conhecimento sobre esse programa, entraves políticos e a necessidade de contemplar a temática nas comissões de trabalho do controle social. Para a superação desses desafios é necessário construir de forma coletiva, com todos os atores sociais (usuários, trabalhadores, gestores e prestadores de serviço) envolvidos no processo de saúde, estratégias que possibilitem mudanças nas relações, nos processos, na saúde e nos indivíduos.

\section{CONSIDERAÇÕES FINAIS}

Ao longo do processo de investigação e pela análise e discussão dos resultados, buscou-se aproximar de uma resposta para o problema de pesquisa e, desse modo, conhecer a compreensão sobre a Educação Permanente na perspectiva dos gestores. Esse entendimento foi respondido de várias formas, contudo, percebeu-se que a compreensão dos entrevistados ainda é muito incipiente e por isso a Educação Permanente é compreendida como sendo algo importante para mudanças necessárias no processo de formação dos profissionais, mas dentro dos propósitos da Educação Continuada.

Ao analisar os principais desafios para a implementação da PEPS, na concepção dos coordenadores de serviço, percebe-se que esses são reflexos do modo de gestão vigente, centralizado e hierarquizado. Assim, a Educação Permanente em Saúde torna-se um importante instrumento capaz de contribuir na mudança do cenário e garantir aos trabalhadores e usuários o seu papel de protagonistas do sistema de saúde.

Salienta-se a importância dos coordenadores buscarem conhecimento sobre a PEPS com vistas a mudanças nas práticas de saúde do município e nas práticas de formação dos profissionais. Um maior envolvimento de todos os atores envolvidos no processo de saúde qualificará o atendimento as demandas da população. Enfatiza-se a necessidade das instituições de ensino investir na mudança do perfil dos futuros profissionais de saúde com vistas ao SUS.

\section{REFERÊNCIAS}

1. Lopes RS, Piovesan ETA, Melo LO, Pereira MF. Potencialidades da educação permanente para a transformação das práticas em saúde. Comun. Cienc. Saúde. 2007;18(2):147-55.

2. Ministério da Saúde (BR). Secretaria de Gestão do Trabalho e da Educação na Saúde. Departamento de Gestão da Educação em Saúde. Política Nacional de Educação em saúde. Brasília; 2009.

3. Ministério da Saúde (BR). Portaria GM/MS no 1.996, de 20 de agosto de 2007. Dispõe sobre as diretrizes para a implementação da Política Nacional de Educação 
Permanente em Saúde e dá outras providências [Internet]. Brasília; 2007 [acesso em 3 abr 2012]. Disponível: http://portal.saude.gov.br/portal/arquivos/ pdf/Portaria_1996-de_20_de_agosto-de-2007.pdf

4. Mancia JR; Cabral LC; Koerich MS. Educação permanente no contexto da enfermagem e na saúde. Rev. bras. enferm. 2004;57(5):605-10.

5. Lopes EM, Anjos SJSB, Pinheiro AKB. Tendência das ações de educação em saúde realizadas por enfermeiros no Brasil. Rev. enferm. UFRJ. 2009;17(2):273-7.

6. Silva LAA da, Franco GP, Leite MT, Pinno C, Lima VML, Saraiva N. Concepções educativas que permeiam os planos regionais de educação permanente em saúde. Texto Contexto Enferm. 2011;20(2):340-8.

7. Ceccim RB. Educação permanente em saúde: descentralização e disseminação de capacidade pedagógica na saúde. Cienc. saude colet. 2005;10(4):975-66.

8. Ministério da Saúde (BR). Secretaria de Gestão do Trabalho e da Educação na Saúde. Departamento de Gestão da Educação na Saúde. Curso de formação de facilitadores de educação permanente em saúde: unidade de aprendizagem Trabalho e relações na produção do cuidado. Brasília; 2005.

9. Ceccim RB; Feuerwerker LCM. O Quadrilátero da formação para a área da saúde: ensino, gestão, atenção e controle social. Physis: Revista Saude Colet. 2004;14(1):41-65.

10. Minayo MCS. O desafio do conhecimento: pesquisa qualitativa em saúde. 10ª ed. São Paulo: Hucitec; 2007.

11. Ministério da Saúde (BR). Conselho Nacional de Saúde. Diretrizes e normas regulamentadoras de pesquisas envolvendo seres humanos. Resolução n. 196, de 10 de outubro de 1996. Brasília; 1996.

12. Silva LAA, Ferraz F, Lino MM, Backes VMS, Schmidt SMS. Educação permanente em saúde e no trabalho de enfermagem: perspectiva de uma práxis transformadora. Rev. gauch. enferm. 2010;31(3):557-61.

13. Marandola TR, Marandola CMR, Melchior R, Baduy RS. Educação Permanente em Saúde: conhecer para compreender. Espaç. saúde. 2009;10(2):53-60.

14. Ministério da Saúde (BR). Conselho Nacional de Saúde. Princípios e Diretrizes para a NOB/RH-SUS. $4^{\text {a }}$ ed. Brasília; 2002.
15. Ministério da Fazenda (BR). Lei Complementar $\mathrm{n}^{\circ}$ 101, de 4 de maio de 2000. Estabelece normas de finanças públicas voltadas para a responsabilidade na gestão fiscal e dá outras providências [Internet]. Brasília; 2000 [acesso em 3 abr 2012]. Disponível: http://www.tesouro. fazenda.gov.br/legislacao/download/contabilidade/ lei_comp_101_00.pdf

16. Taveira ZZ. Precarização dos Vínculos de Trabalho na Estratégia Saúde da Família: Revisão de Literatura. [Monografia]. Belo Horizonte (MG): Universidade Federal de Minas Gerais; 2010.

17. Machado MFAS, Monteiro EMLM, Queiroz DT, Vieira, NFC, Barroso, MGT. Integralidade, formação de saúde, educação em saúde e as propostas do SUS - uma revisão conceitual. Cienc. saude colet. 2007;12(2):335-42.

18. Ortiz MCL, Ribeiro RP, Garanhani ML. Educação a distância: uma ferramenta para educação permanente de enfermeiros que trabalham com assistência perioperatória. Cogitare enferm. 2008;13(4):558-65.

19. Faria MGA, David HMSL. Enfermagem e educação permanente à distância: o exemplo do projeto telessaúde Brasil, núcleo Rio de Janeiro. Cogitare enferm. 2010;15(4):667-73.

20. Senado Federal (BR). Constituição da República Federativa do Brasil: texto promulgado em 05 de outubro de 1988. Brasília [Internet]; 2010 [acesso em 3 abr 2012]. Disponível: http://www.senado.gov. br/legislacao/const/con1988/CON1988_05.10.1988/ CON1988.pdf 